\title{
INCREASING FORECASTING ACCURACY OF TREND DEMAND BY NON-UNEAR OPIIMIZATION OF THE SMOOTHING CONSTANT
}

\author{
Sudipa Sarker ${ }^{1}$ and Mahbub Hossain ${ }^{2}$ \\ ${ }^{1}$ Department of Industrial and Production Engineering \\ Bangladesh University of Engineering and Technology, Dhaka-1000, Bangladesh \\ ${ }^{2}$ Planning Manager, Renata Limited, Dhaka-1216, Bangladesh \\ *Corresponding email: sudipa@ipe.buet.ac.bd
}

\begin{abstract}
Linear Regression is often used for predicting the initial parameters of the forecasting models. But if the underlying demand model is not linear, linear regression does not produce optimal values of these parameters. Again for a novice user predicting the smoothing constants for level and trend demand forecast is not easy and recommended values of these constants may result in larger forecast errors. In this paper real life data of a pharmaceutical company is used to show that forecasting accuracy greatly improves with the non linear optimization of the smoothing constant. It is done using an EXCEL solver where the solver tries to optimize and find the values of the smoothing constants by minimizing the mean square error (MSE).
\end{abstract}

Key Words: Non-Linear Optimization, Smoothing Constant, Trend Demand.

\section{INTRODUCTION}

The pharmaceutical industry is both blessed and cursed by data. There are a number of data sources and inputs available for Time Series (TS) analysis: total prescriptions, new prescriptions, total units, units by stock keeping unit, units by strength, days of therapy, patient days, retail units, hospital units, sales to wholesalers, retail pharmacy inventories and so forth[1]. In this paper actual sales data of a blockbuster product of a local pharmaceutical industry is used and analyzed by using the EXCEL spreadsheets.

Spreadsheets have long had the ability to perform regression analysis using ordinary least squares (OLS), giving analysts the ability to implement a variety of regression based time series techniques (e.g. linear trend models, polynomial trend models seasonal model using indicator variables etc.). The accessibility and familiarity of spreadsheet interface, along with its modeling and graphing capabilities, makes this the tool of choice for many business analysts [2]. Fortunately, all the major spreadsheet packages now include an optimization tool known as Solver. Solver can be used to maximize or minimize the values of the target worksheet cell (containing any formula) by altering the value of selected changing cells in the spreadsheet that influence the value in the target cell. Solver also allows constraints to be placed on the values of any cells in the worksheet. Thus solver is a general purpose tool capable of solving constrained linear and nonlinear optimization problem [3].

The main idea of the paper revolves around the fact that linear regression does not works well when the data do not properly fit with the least square regression line and optimizing the smoothing constants by using a non linear optimization tool (such as Solver) will not only improve the accuracy of the forecast but also will give better values of initial parameters.

\section{LITERATURE REVIEW}

Smoothing models are very common in TS analysis for their simplicity and ease of use. A variety of smoothing models have been proposed for short range forecasting over the years. The exponential smoothing models reported in the literature have been classified along two dimensions; these are constant smoothing models such as simple exponential smoothing (Holt 1957) [4] or Brown's linear model (1963) [5] and adaptive smoothing models such as Chow (1965) [6] and Trigg and

\begin{tabular}{llll}
\multicolumn{2}{l}{ Nomenclature } & & \\
$x_{t}$ & Actual Demand & $\hat{b}_{t}$ & Trend Estimate \\
$a$ & Level & $\tau$ & Period \\
$b$ & Trend & $\sigma$ & Smoothing Constant for Level \\
$t$ & Time Period & $\beta$ & Smoothing Constant for Trend \\
$\hat{x}_{t}$ & Forecast & MSE & Mean Square Error \\
$\hat{a}_{t}$ & Level Estimate & MAPE & Mean Absolute Percentage Error
\end{tabular}


Leach (1967) [7] where smoothing parameters had been allowed to adapt in response to forecast errors. In this paper the smoothing constant is adaptive in the sense that it is set at the value where forecast errors are minimized but is different than other adaptive models as it doesn't update itself with time. Use of spreadsheets to implement exponential smoothing and other smoothing techniques is becoming a trend in management science textbooks [8, 9, 10,12]. As Microsoft Office gets more and more pervasive, it is not uncommon to use EXCEL Solver to optimize the smoothing constant(s) [3] in exponential smoothing techniques. With the increasing capabilities of Solver, forecasters are encouraged to adopt the idea of optimizing parameters normally treated as constants in most textbooks. A recent paper by Rasmussen goes further to optimize the initial values along with the smoothing constants [11]. Rasmussen also gave a rather detail account of using Solver to optimize the initial values and parameters in Holt's method and Holt-Winter's method. Ragsdale and Plane [3], mentioned the use of spreadsheet solver to simultaneously optimize the regression parameters and seasonal adjustments when regression models are used to forecast TS.

\section{THE TREND MODEL}

This type of plot is very helpful to understand the underlying demand model. The graph depicts an increasing trend. Assuming the trend as linear, the underlying model can be expressed as:

$x_{t}=a+b t$
Where, $a=$ level (intercept) and

$b=$ linear trend (slope), $x_{t}=$ demand in period $\mathrm{t}$

While in Fig. 1, 2008 sales data clearly shows a linear trend model with a value of $\mathrm{R}$ square a.k.a. coefficient of determination of $0.815,2007$ data shows a poor linear fit with $\mathrm{R}$ square value of 0.219 . The two equations on the graph show that both the slope and the intercept are different for two years indicating the usual year to year growth of a mature product in the pharmaceutical industry. To account for this growth factor two years of data are used together.

Fig. 2 shows the combined curve fitted with least square regression line. The graph shows that plotting two years of data together, actually, improved the linear quality of the sales curve, as the $\mathrm{R}$ square value jumps to 0.828 . Again, a curve while fitted with a linear regression line which has an $\mathrm{R}$ square value of 0.828 will seem visually linear and most people will take the actual demand data as a linear trend model.

But in this paper it is demonstrated that for these types of data, a non linear optimization of smoothing constants actually gives an accurate forecast than that of linear regression models.

In this paper two models are used to forecast the sales data. The first method is simple linear regression and the second one is Holt's exponential smoothing method. Two examples of Holt's method are shown; in one smoothing constants are optimized by the Solver and in other recommended values of smoothing constants are used. Two years of historical data are then used to forecast for first three months (one quarter of a year) of the third year and corresponding MSEs are observed.

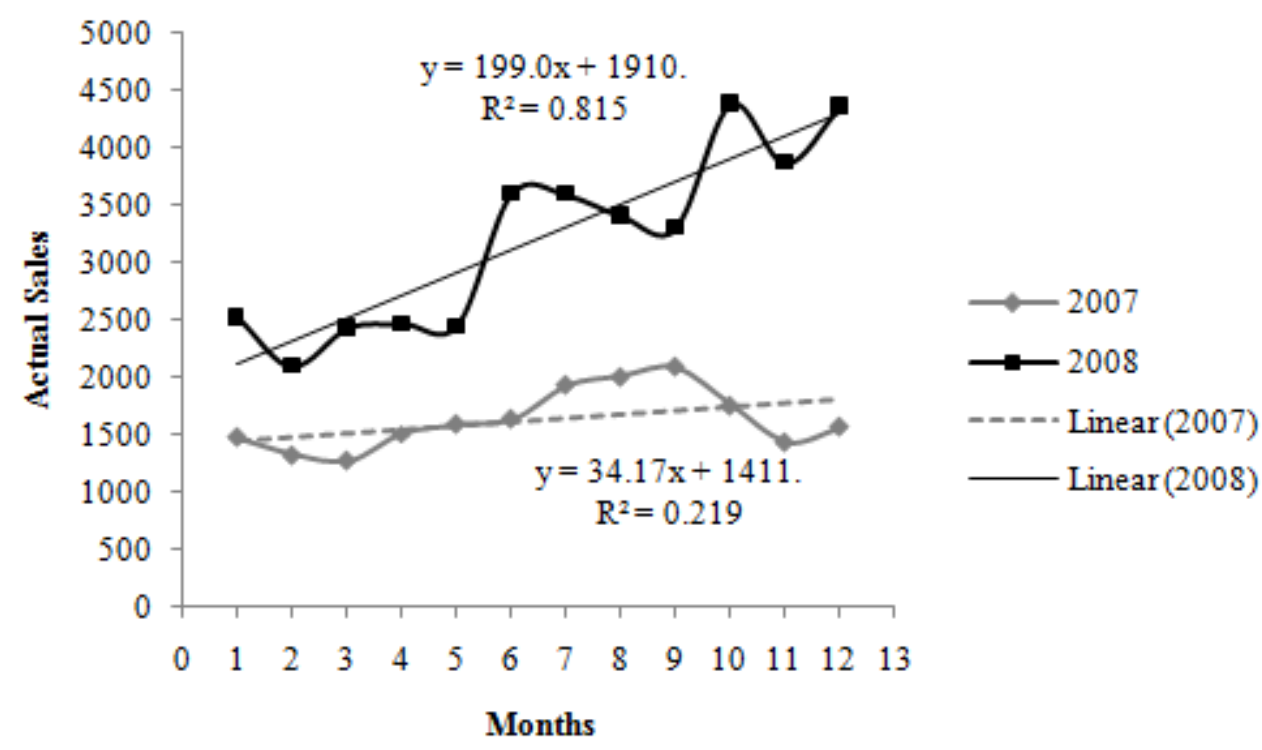

Figure1: Time Series data of an "A" category product 
Forecast based on linear regression can be computed by

$\min _{\hat{a}, \hat{b}}: \mathrm{MSE}$

$\hat{x}_{t}=\hat{a}+\hat{b} t$

where $\hat{a}=$ estimate of level,

$\hat{b}=$ estimate of trend,

$\hat{x}_{t}=$ forecast for period $\mathrm{t}$

$\hat{a}$ and $\hat{b}$ also known as regression parameters and estimated by minimizing the mean square error. and the MSE in (3) is defined as

$\operatorname{MSE}=\frac{1}{\mathrm{n}} \sum_{\mathrm{t}=1}^{\mathrm{n}}\left(x_{t}-\hat{x}_{t}\right)^{2}$

In EXCEL “TREND” function can be used to do the forecast using the simple regression method.

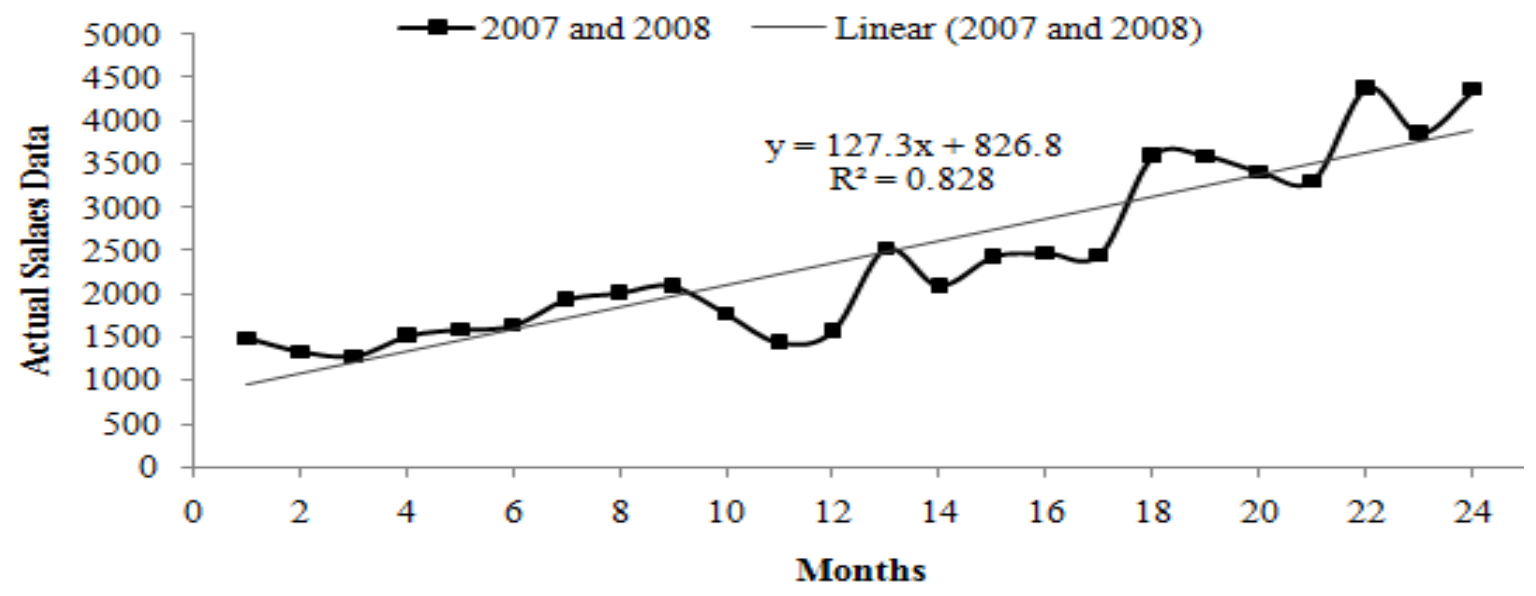

Figure2: Combined sales data of 2007 and 2008 fitted with linear regression lines

Holt's method is more flexible than linear regression since it updates the level and trend parameters, which are constants in linear regression. According to Holt's method, forecast at time $t$ for a period of $t+\tau$ are given by

$\hat{x}_{t+\tau}=\hat{a}_{t}+\tau \hat{b}_{t}$

The level parameter $\hat{a}_{t}$ is updated as

$\hat{a}_{t}=\alpha x_{t}+(1-\alpha) \hat{a}_{t-1}$

The trend parameter $\hat{b}_{t}$ is updated as

$\hat{b}_{t}=\beta\left(\hat{a}_{t}-\hat{a}_{t-1}\right)+(1-\beta) \hat{b}_{t-1}$

$\alpha$ and $\beta$ are the smoothing constants and $0 \leq \alpha, \beta \leq 1$

In Holt's method, initial values of these parameters are needed to update the values of level and trend parameters. These initial values can be measured in various ways. In our case we will take first 5 months data to calculate the values of the initial parameters. We will use the following equations [10]:

$\hat{a}_{0}=\frac{6}{n(n+1)} \sum_{t=-n+1}^{0} t x_{t}+\frac{2(2 n-1)}{n(n+1)} \sum_{t=-n+1}^{0} x_{t}$

$\hat{b}_{0} \frac{12}{n\left(n^{2}-1\right)} \sum_{t=-n+1}^{0} t x_{t}+\frac{6}{n(n+1)} \sum_{t=-n+1}^{0} x_{t}$
Table 1 shows the resultant MSE of simple linear regression performed in EXCEL. The data of 2007 and 2008 is used to forecast for the first four months (Jan-April) of 2009.

Table 2 shows the resultant MSE for Holt's method performed in EXCEL. The solver tries to optimize the parameters $\alpha$ and $\beta$ while minimizing the MSE value. Table 2 also shows the resultant parameters find by the EXCEL solver.

Most of the research on simple exponential smoothing has assumed a range of values between 0 to1 for $\alpha$, although a more restricted range of 0.1-0.3 is typical practice. It is widely held that a more complex model should be entertained if the best value falls above 0.30 during the model fitting process [9]. Brown (1997) recommended $\beta$ in the range of 0.75 ('fast smoothing' for shorter time series) to 0.90 (normal smoothing). In table 3 , resultant MSE is shown for $\alpha$ value of 0.3 and $\beta$ value of 0.75 . The only change made for Table 3 is that smoothing constants are not optimized by the Solver rather it is fixed at recommended values. 
Table 1: Results of Simple Linear Regression

\begin{tabular}{|c|c|c|c|c|c|c|}
\hline Year & Months & Period t & Actual Demand & Forecast & MSE & MAPE \\
\hline \multirow[t]{12}{*}{2007} & Jan & 1 & 1478 & 954.2 & & \\
\hline & Feb & 2 & 1324 & 1081.541304 & & \\
\hline & Mar & 3 & 1276 & 1208.882609 & & \\
\hline & Apr & 4 & 1514 & 1336.223913 & & \\
\hline & May & 5 & 1586 & 1463.565217 & & \\
\hline & Jun & 6 & 1633 & 1590.906522 & & \\
\hline & Jul & 7 & 1933 & 1718.247826 & & \\
\hline & Aug & 8 & 2007 & 1845.58913 & & \\
\hline & Sep & 9 & 2089 & 1972.930435 & & \\
\hline & Oct & 10 & 1758 & 2100.271739 & & \\
\hline & Nov & 11 & 1437 & 2227.613043 & & \\
\hline & Dec & 12 & 1564 & 2354.954348 & & \\
\hline \multirow[t]{12}{*}{2008} & Jan & 13 & 2520 & 2482.295652 & & \\
\hline & Feb & 14 & 2092 & 2609.636957 & & \\
\hline & Mar & 15 & 2430 & 2736.978261 & & \\
\hline & Apr & 16 & 2460 & 2864.319565 & & \\
\hline & May & 17 & 2443 & 2991.66087 & & \\
\hline & Jun & 18 & 3600 & 3119.002174 & & \\
\hline & Jul & 19 & 3592 & 3246.343478 & & \\
\hline & Aug & 20 & 3406 & 3373.684783 & & \\
\hline & Sep & 21 & 3302 & 3501.026087 & & \\
\hline & Oct & 22 & 4379 & 3628.367391 & & \\
\hline & Nov & 23 & 3864 & 3755.708696 & & \\
\hline & Dec & 24 & 4360 & 3883.05 & 183026.4551 & $15.39 \%$ \\
\hline \multirow[t]{3}{*}{2009} & Jan & 26 & 4425 & 4137.732609 & 614490.8338 & $12.20 \%$ \\
\hline & Feb & 27 & 4613 & 4265.073913 & & \\
\hline & Mar & 28 & 5673 & 4392.415217 & & \\
\hline
\end{tabular}


Table 2: Results of Non-Linear Optimization of Smoothing Constant

\begin{tabular}{|c|c|c|c|c|c|c|c|c|c|}
\hline Year & Months & Period $t$ & Actual Demand & Level & Trend & Forecast & MSE & MAPE & \\
\hline \multirow[t]{5}{*}{2007} & Jan & 1 & 1478 & & & - & & \multirow[b]{5}{*}{$13.03 \%$} & \\
\hline & Feb & 2 & 1324 & & & - & & & \begin{tabular}{l|l}
$\alpha$ & 0.371
\end{tabular} \\
\hline & Mar & 3 & 1276 & & & - & & & \begin{tabular}{l|l}
$\boldsymbol{\beta}$ & 0.218
\end{tabular} \\
\hline & Apr & 4 & 1514 & & & - & & & \\
\hline & May & 5 & 1586 & 1516 & 40.6 & - & 182463 & & \\
\hline \multirow{19}{*}{2008} & Jun & 6 & 1633 & 1584.94 & 46.79 & 1556.60 & & & \\
\hline & Jul & 7 & 1933 & 1743.46 & 71.18 & 1631.72 & & & \\
\hline & Aug & 8 & 2007 & 1885.98 & 86.76 & 1814.64 & & & \\
\hline & Sep & 9 & 2089 & 2015.86 & 96.17 & 1972.75 & & & \\
\hline & Oct & 10 & 1758 & 1980.73 & 67.51 & 2112.04 & & & \\
\hline & Nov & 11 & 1437 & 1821.54 & 18.01 & 2048.24 & & & \\
\hline & Dec & 12 & 1564 & 1737.35 & -4.31 & 1839.55 & & & \\
\hline & Jan & 13 & 2520 & 2024.91 & 59.42 & 1733.05 & & & \\
\hline & Feb & 14 & 2092 & 2087.18 & 60.04 & 2084.33 & & & \\
\hline & Mar & 15 & 2430 & 2252.10 & 82.94 & 2147.22 & & & \\
\hline & Apr & 16 & 2460 & 2381.38 & 93.06 & 2335.04 & & & \\
\hline & May & 17 & 2443 & 2462.78 & 90.51 & 2474.44 & & & \\
\hline & Jun & 18 & 3600 & 2941.50 & 175.28 & 2553.30 & & & \\
\hline & Jul & 19 & 3592 & 3293.02 & 213.76 & 3116.77 & & & \\
\hline & Aug & 20 & 3406 & 3469.40 & 205.60 & 3506.78 & & & \\
\hline & Sep & 21 & 3302 & 3536.66 & 175.39 & 3675.00 & & & \\
\hline & Oct & 22 & 4379 & 3959.41 & 229.40 & 3712.06 & & & \\
\hline & Nov & 23 & 3864 & 4068.35 & 203.10 & 4188.81 & & & \\
\hline & Dec & 24 & 4360 & 4304.29 & 210.27 & 4271.44 & & & \\
\hline \multirow[t]{3}{*}{2009} & 1 & 26 & 4425 & & & 4514.56 & 188344 & $5.82 \%$ & \\
\hline & 2 & 27 & 4613 & & & 4724.82 & & & \\
\hline & 3 & 28 & 5673 & & & 4935.09 & & & \\
\hline
\end{tabular}


Table 3: Results of Halts Method Using Recommended Smoothing Parameters

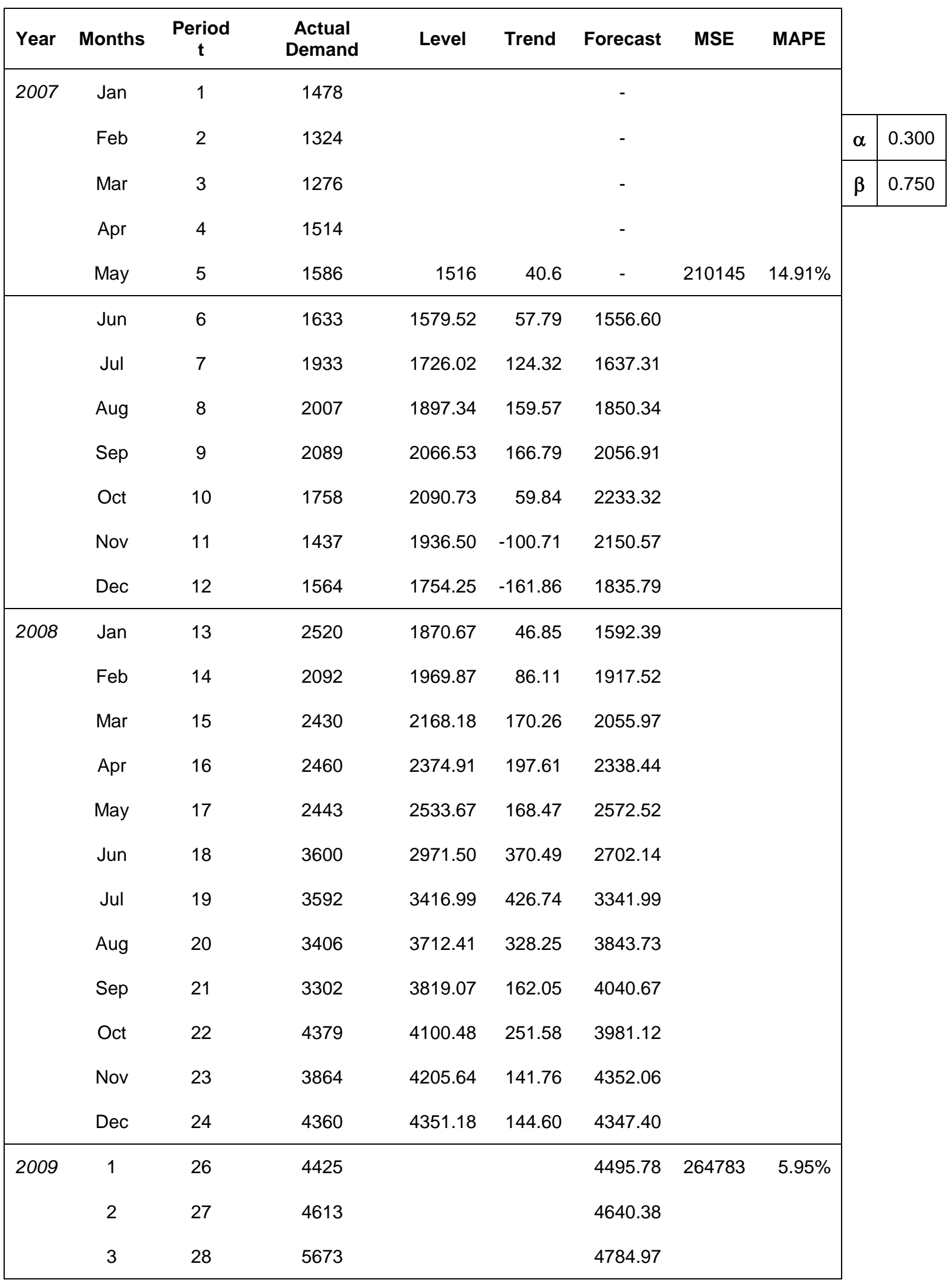




\section{RESULTS AND DISCUSSION}

Comparing three resultant MSEs, it is clear that MSE of Holt's method using non linear optimization of smoothing constant is less than MSEs of other two methods. It proves that for a seemingly linear demand trend a non linear optimization of smoothing constant outperforms linear regression method. But it is also evident that the MSE of linear regression method is not that different from the Holt's method. This happened because combining two years of sales data actually improved the linearity of the sales curve and hence linear regression worked well. For a strict linear model having an $\mathrm{R}$ square value above 0.9 linear regression might outperform the non linear optimization method.

The choice between the recommended and optimized values of the constant is obvious as the later clearly outperforms the former. So instead of choosing the constants arbitrarily or selecting a value from many trial and error solutions it is better to use software that optimizes the smoothing constant.

Here we compared the three methods using MSE as the basis. The comparison can also be done using Mean Absolute Deviation (MAD) or Mean Absolute Percentage of Error (MAPE) as the basis. Interestingly MSE value may increase when the solver tries to optimize the MAPE.

\section{CONCLUSIONS}

This paper outlines an approach whereby a nonlinear optimization of smoothing constant for a seemingly linear demand gives better values of initial parameters and smoothing constants than the linear regression method. It also relieves the user from choosing an arbitrary constant which may or may not give an accurate forecast. Though solver offered a better result in this case, we must also take into account the fact that for different starting values of $\alpha$ and $\beta$, solver may show different MSE values. So it is necessary to run a check on the model by choosing different starting values of smoothing constants. Fortunately, in our example that was not the case. The model is checked for different starting values of $\alpha$ and $\beta$ and the resultant MSE was same for all of them.

\section{REFERENCES}

[1] C. J. Arthur, Forecasting for the Pharmaceutical Industry, Gower Publishing Limited; 2006, p.73

[2] Camm, JD, Evans JR, Management science and decision technology, $1^{\text {st }}$ ed. Cincinnati, $\mathrm{OH}$ : Southwestern College Publishing; 2000. p.390

[3] Ragsdale T. C. , Plane R. D. 2000, On modeling time series data using spreadsheets, OMEGA The International Journal of Management Science 28 pp. 215-221

[4] Ragsdale, CT. Spreadsheet modeling and decision analysis, $3^{\text {rd }}$ ed. Cincinnati, $\mathrm{OH}$ : Southwestern College Publishing; 2001. p. 94.

[5] Render B, Stair RM. Quantitative analysis for management, $7^{\text {th }}$ ed. Englewood Clils, NJ: Prentice-Hall; 2000.

[6] Winston WL, Albright CS. Practical management science, $2^{\text {nd }}$ ed. Paci/c Grove, CA: Duxbury; 2001. p. 953.

[7] Makridakis, SG, Wheelwright SC, Hyndman RJ. Forecasting methods and applications, $3^{\text {rd }} \mathrm{Ed}$. New York: John Wiley \& Sons

[8] Rasmussen R 2004, On time series analysis and optimal parameters, OMEGA The International Journal of Management Science vol 32, pp. 11120.

[9] Everette S. G. Jr. 1985, Exponential Smoothing: The state of Art, Journal of Forecasting Vol.4 pp. 1-28

[10] Silver A. E., Pyke F. D. Peterson R. 1998, Inventory Management and Production Planning and Scheduling, $3^{\text {rd }}$ Ed. New York: John Wiley \& Sons 